LA-UR-00-6046

Approved for public release; distribution is unlimited.

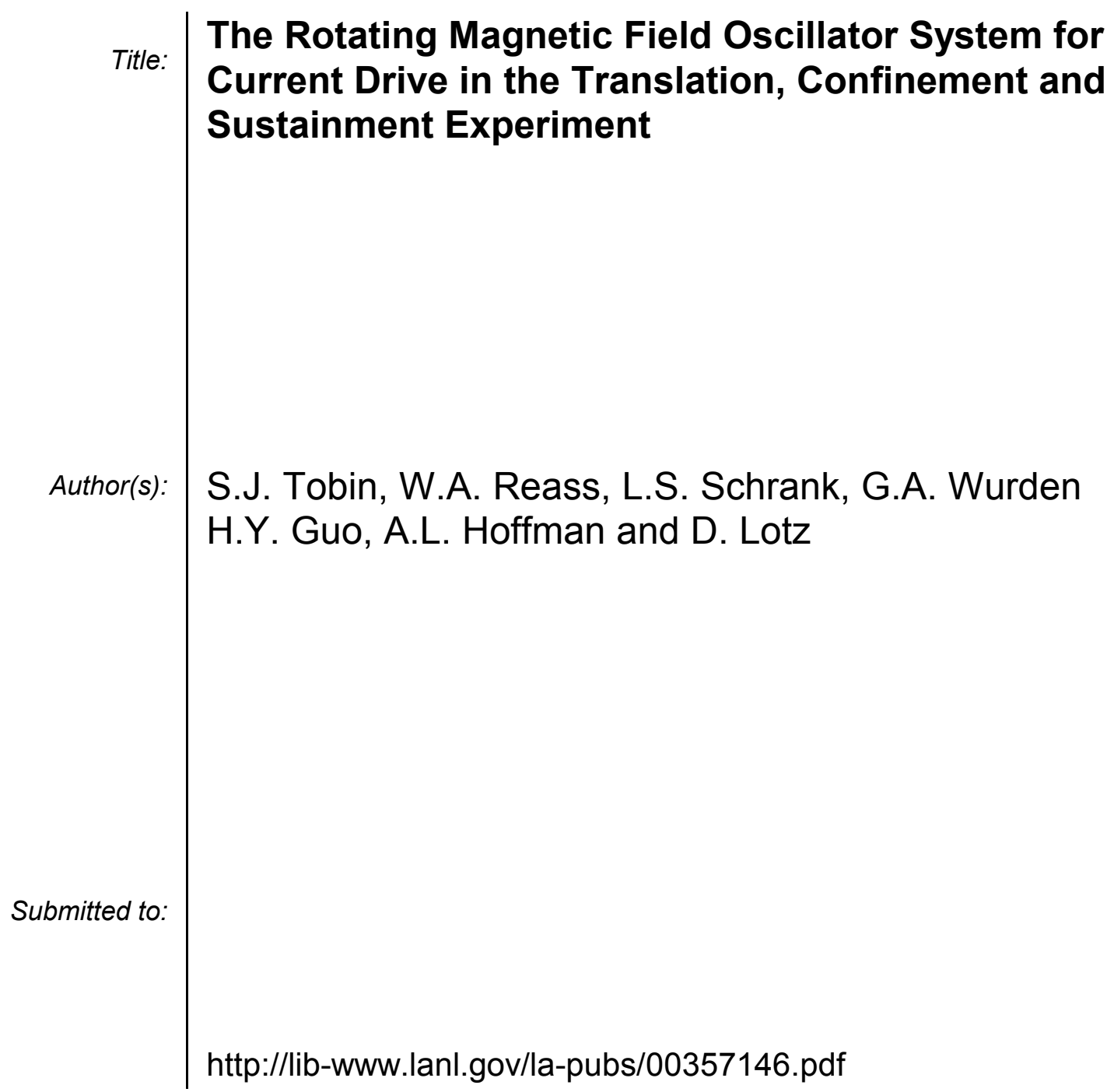

Los Alamos National Laboratory, an affirmative action/equal opportunity employer, is operated by the University of California for the U.S. Department of Energy under contract W-7405-ENG-36. By acceptance of this article, the publisher recognizes that the U.S. Government retains a nonexclusive, royaltyfree license to publish or reproduce the published form of this contribution, or to allow others to do so, for U.S. Government purposes. Los Alamos National Laboratory requests that the publisher identify this article as work performed under the auspices of the U.S. Department of Energy. Los Alamos National Laboratory strongly supports academic freedom and a researcher's right to publish; as an institution, however, the Laboratory does not endorse the viewpoint of a publication or guarantee its technical correctness. 


\title{
The Rotating Magnetic Field Oscillator System for Current Drive in the Translation, Confinement and Sustainment Experiment
}

\author{
S.J. Tobin, W.A. Reass, L.S. Schrank and G.A. Wurden \\ Los Alamos National Laboratory, Los Alamos, NM 87545 \\ H.Y. Guo, A.L. Hoffman and D. Lotz \\ University of Washington, Redmond Plasma Physics Laboratory, Seattle, Washington 98052
}

(Received Jan., 2001; accepted for publication March 22, 2001)

The experimental setup and test results for the $\sim 125 \mathrm{MW}$ rotating magnetic field current drive system of the Translation, Confinement and Sustainment Experiment at the University of Washington are described. The oscillator system, constructed at Los Alamos National Laboratory, drives two tank circuits $\left(15 \mathrm{kV}_{\text {peak }}\right.$ potential, $8.5 \mathrm{kA}_{\text {peak }}$ maximum circulating current in each tank to date) operated $90^{\circ}$ out of phase to produce a $54 \mathrm{G}$ rotating magnetic field with a frequency of $163 \mathrm{kHz}\left(\omega=1.02^{\times} 10^{-6}\right.$ $\left.\mathrm{s}^{-1}\right)$. Programmable waveform generators control "hot deck" totem pole drivers that are used to control the grid of 12 Machlett 8618 magnetically beamed triode tubes. This setup allows the current to be turned on or off in less than $100 \mathrm{~ns}\left(\sim 6^{\circ}\right)$. Both tank circuits are isolated from the current source by a 1:1 air core, transmission line transformer. Each tank circuit contains two saddle coils (combined inductance of $1.6 \mu \mathrm{H})$ and radio frequency capacitors $(580 \mathrm{nF})$. Test results are presented for three conditions: no external load, a resistive external load and a plasma load. A SPICE model of the oscillator system was created. Comparisons between this model and experimental data are given.

\section{INTRODUCTION}

The Translation, Confinement and Sustainment (TCS) Experiment located at the Redmond Plasma Physic Laboratory (RPPL) of the University of Washington was designed to apply, for the first time, a rotating magnetic field (RMF) to a high-temperature field reversed configuration (FRC) plasma $\left(\mathrm{T}_{\mathrm{e}} \sim 100\right.$ $\mathrm{eV}$ and $\mathrm{T}_{\mathrm{i}} \sim 300 \mathrm{eV}$ ) [1]. The RMF current drive technique is a special case of the more general $\mathbf{j} \mathbf{x} \mathbf{B}$ current drive scheme, by which electrons are "pulled" along with the rotating magnetic field [2]. Unlike inductive current drive systems, RMF current drive is steady state. The RMF is used for the purpose of building up and sustaining the magnetic flux of the FRC. The experimental goal is to benchmark this current drive technique against theoretical predictions, and to determine the robustness of the FRC plasma to this external perturbation. An initial goal for the RMF system was to extend the lifetime of the FRC by a factor of $\sim 3$; this goal has already been achieved with pulses lasting $1 \mathrm{~ms}$. It was anticipated that a $1 \mathrm{~ms}$ pulse would become limited by particle inventory rather than resistive flux losses. At present particle inventory is not limiting lifetime, exactly why is not yet understood.

This article describes the highest power RMF system ever built. It is a rugged, versatile system, which was constructed and tested at Los Alamos National Laboratory (LANL). Programmable waveform generators produce the waveforms that control the high power, magnetically beamed triode tubes. These tubes drive current in the primary of a 1:1 transformer, while the secondary of the transformer is connected in parallel with a tank circuit for which the RMF antennas are the inductor. Some other RMF system include the Rotamak system at Flinders University [3] which utilized a lower power oscillator and conventional vacuum tube technology and the Star Thrust Experiment at the University of Washington which is lower in power than TCS and utilized IGBTs to switch the current instead of triodes [4].

\section{EXPERIMENTAL SETUP}

At RPPL the LANL built system was interfaced with the TCS tank circuit. The oscillator system has two halves, each of which was fabricated so that the inductance and capacitance are equal. The current in the antennas of the two halves is driven at the same resonant frequency. The two antenna systems (each system has 2 antennas) are driven $90^{\circ}$ out of phase so that the sum of the magnetic fields from the two antenna systems will be a RMF that rotates at the driven frequency. A simplified circuit diagram of one half of the oscillator is depicted in Fig. 1. Note that the ignitron, hard dump, soft dump and main capacitor bank 
are actually shared by the two halves of the oscillator. For future reference the two halves will be referred to as horizontal and vertical, these labels refer to the direction of the magnetic field produced by the respective antenna systems.

To simplify the discussion of the experimental system, unless stated otherwise, the following discussion applies to one tank circuit (i.e. one antenna system which contains two antennas). A 1:1 transformer electrically isolates the current source from the tank circuit. On the primary side of the transformer, the fundamental components are the triodes, the main capacitor bank and the transformer. The energy needed to generate the magnetic field comes from the main capacitor bank, which has a maximum capacitance of $950 \mu \mathrm{F}$. Depending on the pulse length of interest, only a fraction of this energy storage capacity is used. The six Machlett 8618 magnetically beamed triodes, connected in parallel, are used to switch the current on and off. The secondary of the transformer is connected in parallel with the radio frequency (RF) capacitors $(580 \mathrm{nF})$ and saddle coils (combined inductance of $1.6 \mu \mathrm{H})$ of a tank circuit.

About a minute before a shot is to be initiated, the power supply, connected in parallel with the main capacitor bank and the radio frequency bypass capacitor, charges both capacitors to the operating potential, up to $20 \mathrm{kV}$. The bypass capacitor is needed to give the desired time response. For this reason the bypass capacitor is located as close as possible to the transformer. The $0.5 \Omega$ resistors connected in series with the respective capacitors are there to limit the current in case of a fault condition.

Before a shot, a waveform generator (Model 39 Wavetek) is programmed independently by means of a LabVIEW control program. This program calls an Excel spreadsheet that contains a Visual Basic macro, which calculates the value of each addresses of the waveform generator. The clocks of both waveform generators are shared. The waveform generators give excellent time control. The minimum possible interval for a single address is $33 \mathrm{~ns}$. The operator can vary both the duration of each individual pulse within the pulse train as well as the frequency between pulses within the pulse train.

When a trigger is received from the experiment controller, a waveform generator produces a train of voltage pulses. These voltage pulses are transferred into optical signals thus keeping the waveform generator, which is located in the control room, isolated from the experiment. The optical signal controls the potential of the grid of several triodes (Eimac 3CPX1500A7) located on two hot decks named "on" and "off." The term "hot" was given since the metal cases of these decks float relative to ground. One "on" deck utilizes six Eimac triodes and one "off" deck utilizes four Eimac triodes. The two decks are connected in a totem pole configuration to the grids of the six, 8618 Machlett triodes so that the Machlett tubes will turn on and off in unison. The default state of the hot decks is with the "off" deck biasing the Machlett grid to $-2 \mathrm{kV}$; thus, keeping current from flowing through the Machlett tubes.

The $8 \Omega$ resistor located in between the decks and the grid of the 8618 tubes is there to damp out parasitic resonances that can cause the grid potential of the 8618 to parasitically oscillate. With the spurious resonances controlled, the 8618 tubes to turn on and off in a clean fashion as the potential of the grid varies between $+4 \mathrm{kV}$ and $-2 \mathrm{kV}$, respectively. Both the $1 \Omega$ resistor (connected between the Machlett tubes and the transformer) as well as the snubbers (connected between the plate of the tubes and ground, $3 \Omega$ resistor and $17 \mathrm{nF}$ capacitor) are necessary to suppress resonant frequencies other than that at which the circuit is being driven. Also, the snubber circuit damps out unwanted ringing from stray inductance originating in the cables, transformer, load, etc.

Now, addressing the protective circuitry, a current monitor (not shown) connected in series with each of the Machlett tubes, detects if an arc occurred. The excessively large current of such an event can damage a tube if it is not eliminated within 5 to $10 \mu \mathrm{s}$. In response to the detection of a large current, the ignitron is fired by the protective circuitry, essentially shorting the capacitor bank. In such a fault condition, the $0.5 \Omega$ current-limiting resistor located on the capacitor bank is responsible for dissipating the energy. The soft and hard dumps, located in parallel with the capacitors, provide the means of discharging the energy of the capacitors under non-fault conditions. The soft dump is automatically triggered one second after the shot, followed a few seconds later by the hard dump. 
Completing the discussion of the oscillator circuit, the transformer is an efficient, transmission line style, 1:1 transformer with near unity coupling as the leakage inductance is only the unit length inductance of the coaxial cable. The magnetized inductance is that of a single layer solenoid. It provides electrical isolation between the current source and the resonant circuit. The current in the secondary side feeds energy into the resonant LC circuit where the inductor is the pair of saddle coils (combined inductance of $1.6 \mu \mathrm{F}$ ). Since losses are low between the antennas and RF capacitors $(\mathrm{Q} \sim 70)$, the circulating current becomes rather large $(8.5 \mathrm{kApp}$, bank potential $=15 \mathrm{kV}$, no load, driven on resonance). The six Machlett tubes can contribute $1.5 \mathrm{kA}$ per pulse to the resonating tank circuit. The tank circuit losses and the tube parameters provide an overall limit to the circulating current.

\section{TEST RESULTS}

In figure 2, signals from different components of the oscillator system are shown. The experimental conditions were the following: bank potential of $10 \mathrm{kV}$, non-loaded circuit driven at the resonant frequency of $163 \mathrm{kHz}$. The tubes were turned on for $40^{\circ}$ on the first period followed by $40^{\circ}$ on the second; for subsequent pulses, the width gradually increases as follows: $60^{\circ}, 80^{\circ}, 110^{\circ}, 135^{\circ}, 165^{\circ}$. Until the end of the waveform, the individual pulse width was maintained at $165^{\circ}$. This gradual ramping up is necessary since the impedance of the tank circuit is initially very low and augments as the circulating current increases. The lower limit of $40^{\circ}$ was determined experimentally since below $40^{\circ}$ the tubes did not turn off fast enough. This slow turn off allowed the parasitic oscillations of the grid potential to cause the Machlett tubes to switch on or off rapidly.

Fig. 2a depicts the waveform generator output, a TTL signal between 0 and 4 volts. Fig $2 \mathrm{~b}$ illustrates the voltage of the hot decks that drive the grid of six Machlett tubes. The potential of the grid is initially -2 $\mathrm{kV}$ so that the Machlett tubes will not conduct. Then the grid potential is driven to $+2.7 \mathrm{kV}$ in $100 \mathrm{~ns}$. The bias potential of the "on" deck $(+4.0 \mathrm{kV})$ is not reached due to the grid current coming from the cathode of the Machlett tube. An unwanted resonance in the grid potential is observable between pulses. The $8 \Omega$ resistor minimizes this resonance to a tolerable level.

In Fig. 2c the tube current for one of the Machlett tubes is illustrated. The depicted maximum current is $\sim 230 \mathrm{~A} /$ tube, a value which is very sensitive to the voltage across the tubes filament which is $\sim 7.5 \mathrm{~V}$. The maximum tube current per tube is $\sim 250 \mathrm{~A}$. During the last three pulses shown, the tube turns off in the middle of the positive plateau. This only occurs when the oscillator is driven without a load. Without a load, the potential of the plate drops below the potential of the grid and the cathode. This causes the current to no longer flow through the tube. In Fig $2 \mathrm{~d}$, it can be seen that the plate potential goes negative around 53 and $59 \mu \mathrm{s}$. Since the cathode is at ground and the grid has a positive potential when turned on, current will not flow from the cathode to the plate.

When the Machlett tube first conducts electrons, it pulls the pre-shot potential of the plate (anode) down from $10 \mathrm{kV}$. Furthermore, this first pulse of current in the primary of the transformer starts the tank circuit oscillating. Since the driving frequency of the Machlett tubes and the resonant frequency of the tank are the same, $180^{\circ}$ after the current flows in one direction in the secondary (and primary), it will flow back in the opposite direction as the energy oscillates between the RF capacitor and the antennas. This feedback from the tank circuit, along with the main bank acting to return the plate to its original potential, cause the potential of the plate to rise above the potential it had at the beginning of the shot. With each subsequent pulse of the tubes, the plate potential (Fig. 2d) gets pulled a little further negative, the current in the tank circuit gets larger so that the plate's potential swings further above and below its pre-shot level $(10 \mathrm{kV}$ in this case). This feedback continues so that in steady state the potential of the plate varies between zero and twice the bank potential ( 0 and $20 \mathrm{kV}$ for an ideal circuit with a $10 \mathrm{kV}$ bank). Due to the parasitic resonances, the experimental system varies between -3 and $+26 \mathrm{kV}$. Since the antennas are isolated by the transformer and since they have a pre-shot potential of ground, the corresponding voltage in the tank circuit swing from $-10 \mathrm{kV}$ to $+10 \mathrm{kV}$ (Fig. 2e). The steady state circulating current of the tank circuit (Fig. 2f) for the horizontal or vertical oscillator was $7.0 \mathrm{kA}_{\text {peak }}(10 \mathrm{kV}$ bank). It is worth noting that the voltage and current in figures (2e) and (2f) are very smooth sinusoidal functions. When the bank potential is set to 15 $\mathrm{kV}$, the circulating current rises to $8.5 \mathrm{kA}_{\text {peak }}$ (no load present) this corresponds to $54 \mathrm{G}$ RMF. The system 
was designed to operate at a maximum bank voltage of $20 \mathrm{kV}$; operating at this potential is expected to produce a RMF of $64 \mathrm{G}$ when not loaded and $54 \mathrm{G}$ if loaded.

In Fig. 3, the current in a Machlett tubes is depicted for a loaded and a non-loaded case. In the loaded case, the potential across the tube remains positive. The non-loaded case is for the same shot as depicted in Fig. 2c. As was stated earlier, the tube stops conducting in the non-loaded case since the potential of the plate goes negative relative to the cathode.

In Fig. 4 the strength of the RMF for a $500 \mu$ s pulse, $10 \mathrm{kV}$ bank, and non-loaded condition is illustrated. Note that it takes about $50 \mu \mathrm{s}$ ( 7 pulses) for the tank circuit to pump up. The standard experimental pulse width is presently $1 \mathrm{~ms}$ and will be increased soon to $20 \mathrm{~ms}$. The only limit to the pulse length at the moment is the waveform generator memory. The quality factor $(\mathrm{Q})$ of the non-loaded circuit was measured from the ring down of the current to be $\sim 70$.

In Fig. 5 the circulating current in the vertical antenna as a function of frequency is illustrated for two conditions: with and without plasma. The introduction of the plasma has two prominent effects: (1) it shifts the resonant frequency from $163 \mathrm{kHz}$ to $172 \mathrm{kHz}$, and (2) it reduces the $\mathrm{Q}$ of the circuit. The resonant frequency of the tank circuit is given by $\omega=\left(\mathrm{L}^{*} \mathrm{C}\right)^{-1 / 2}$. The shift to higher frequency is expected since the plasma decreases the total inductance of the tank circuit.

From Fig. 5 the inductance of the plasma can be estimated. When there is no plasma, the effective inductance of the tank circuit is $1.64 \mu \mathrm{H}\left(\omega=2 * \pi * 163,000 \mathrm{~s}^{-1}\right.$ and $\left.\mathrm{C}=580 \mathrm{~F}\right)$, while with the plasma it is $1.48 \mu \mathrm{H}\left(\omega=2 * \pi * 172,000 \mathrm{~s}^{-1}\right.$ and $\left.\mathrm{C}=580 \mathrm{~F}\right)$. Since the plasma is effectively an inductor connected in parallel with the antennas, the inductance of the plasma is $\sim 15 \mu \mathrm{H}$; this is the parallel inductance necessary to shift the inductance of the tank circuit by $0.16 \mu \mathrm{H}(1.64 \mu \mathrm{H}-1.48 \mu \mathrm{H})$.

\section{MODELING}

An accurate SPICE mode of the oscillator system has been produced and is illustrated in Fig. 6. The primary components in the circuit were described along with Fig. 1. To make the model more accurate, estimates of the inductance and resistance of cables and connections have been included. Furthermore, the coupling coefficient of the transformer was taken to be 0.99 . The $0.5 \mu \mathrm{H}$ located in the tank circuit represent the transformer connections and stray inductance. The $1.5 \mu \mathrm{H}$ on the primary side of the transformer includes the transformer connections too but also the stray inductance of the larger primary circuit. The most challenging aspect of producing this model was accurately modeling the Machlett tubes. This was accomplished by using a fifth order polynomial to characterize the voltage across the tube as a function of current through them. The $1.7 \mathrm{~m} \Omega$ resistor connected in series with the tubes serves as a current monitor for the tubes. When the switch to the left of "Rtube" closes, as determined by positive pulses to the grid of the tubes, current starts to flow in the tubes. The voltage drop across the tubes is given by the following polynomial: $\mathrm{V}=1-2.38^{\mathrm{x}} \mathrm{I}_{\text {tube }}+3.15^{\mathrm{x}} \mathrm{I}_{\text {tube }}{ }^{2}-2.02^{\mathrm{x}} \mathrm{I}_{\text {tube }}{ }^{3}+0.59^{\mathrm{x}} \mathrm{I}_{\text {tube }}{ }^{4}-0.06^{\mathrm{x}} \mathrm{I}_{\text {tube }}{ }^{5}$. The model was tested at several frequencies for both loaded and unloaded cases. For these test cases the voltage across the antennas and tubes, as well as the current through these components, were found to agree reasonably well with experimental measurements.

In Figures 7 and 8 modeling and experimental results (horizontal antenna) are shown for a bank potential of $10 \mathrm{kV}$. In Fig. 7 the antenna current and voltage are both illustrated as a function of the driving frequency. The lines represent the modeling results (solid for voltage and dashed for current) and the individual points are experimental (triangles for voltage and squared for current). The experimental and modeling results are in general agreement, particularly near the resonant operating regime. In Fig. 8 the impact of loading on the antenna current and voltage are depicted. The experimental data points for a conductance of 0.05 siemens were obtained by connection a $20 \Omega$ resistor in parallel with the antennas. The amount that the voltage and current are reduced with increasing load is reasonably reproduced. Fig. 8 is useful for estimating the conductance of the plasma. The conductance of the plasma for a typical shot $\left(\mathrm{n}_{\mathrm{e}}\right.$ $\sim 6^{\mathrm{x}} 10^{18} \mathrm{~m}^{-3}$ and $\mathrm{T}_{\mathrm{e}} \sim 30 \mathrm{eV}$ ) is around 0.05 siemens. This was determined from Fig. 8 and the 
experimental current and voltage values of $4.2 \mathrm{kA}_{\text {peak }}$ and $8.0 \mathrm{kV}_{\text {peak }}$ that were measured for a $10 \mathrm{kV}$ bank plasma shot.

\section{PLASMA RESULTS}

The goal of the TCS experiment is to form a hot $\left(\mathrm{T}_{\mathrm{e}} \sim 100 \mathrm{eV}, \mathrm{T}_{\mathrm{i}} \sim 300 \mathrm{eV}\right.$, maximum $\mathrm{n}_{\mathrm{e}}=2^{\mathrm{x}} 10^{20}$ $\mathrm{m}^{-3}$ ) FRC utilizing the field reversed theta pinch formation technique, translate this FRC to a confinement chamber where the RMF will be utilized to sustain the flux [1]. The major focus of experimental operation to date has been on forming and sustaining cold $\left(\mathrm{T}_{\mathrm{e}} \sim 35 \mathrm{eV}, \mathrm{T}_{\mathrm{i}} \sim 4 \mathrm{eV}\right)$, low density $\left(\mathrm{n}_{\mathrm{e}} \sim 1^{\mathrm{x}} 10^{19} \mathrm{~m}^{-3}\right)$ FRCs in the confinement chamber. This has been achieved by applying the RMF to a pre-ionized gas (15 psi gas puff). A major success of these early experiments is that the FRC is sustained, as long the RMF remains running ( $1 \mathrm{~ms}$ maximum to date).

In Fig. 9 typical plasma data is illustrated for a $12.5 \mathrm{kV}$ bank potential. In Fig. 9a a peak circulating current of $7.4 \mathrm{kA}_{\text {peak }}(\mathrm{RMF}$ strength of $47 \mathrm{G})$ and a steady-state value of $5.7 \mathrm{kA}_{\text {peak }}(\mathrm{RMF}=36 \mathrm{G})$ are shown. The peak in the RMF field strength $(\sim 80 \mu \mathrm{s})$ is due to the combination of the driving frequency varying and the introduction of the plasma. The tank circuit is initially driven at the resonant frequency of the non-loaded, tank circuit (163 kHz). During the first $200 \mu$ s the driving frequency is gradually shifted to the resonant frequency of the circuit when the plasma is present. In Fig. 5 a resonant frequency of $172 \mathrm{kHz}$ was observed; however, $169 \mathrm{kHz}$ is used in the experiment since the peak is broad and it requires less time to shift to $169 \mathrm{kHz}$. An additional note, from the current ring down of Fig. 9a, it was determined that the $\mathrm{Q}$ of the circuit is $\sim 20$ with the plasma present.

The data from Figures $9 \mathrm{~b}$ and $9 \mathrm{c}$ indicate that an FRC was formed. The upper trace (dashed line) in $9 \mathrm{c}$ indicates that the magnetic field strength in the edge of the chamber, in the region outside of the FRC, increased in strength from $40 \mathrm{G}$ to a peak of $150 \mathrm{G}$ before reaching a steady-state value of $100 \mathrm{G}$. The increased magnetic field is necessary to support the plasma pressure exerted by the FRC. The lower trace (solid line) indicates that the magnetic field on the axis of the machine was reversed in direction from a positive $40 \mathrm{G}$ to a peak negative value of $200 \mathrm{G}$ before reaching a steady-state value of $-100 \mathrm{G}$. Field reversal on axis is the signature of a FRC. Fig. $9 \mathrm{~b}$ indicates the magnitude of the flux excluded by the FRC. This indicated the strength of the magnetic pressure that the open field lines just outside the separatrix must support. In figures $9 \mathrm{~d}$ and $9 \mathrm{e}$ the electron temperature and density are illustrated. The electron temperature peaked at $120 \mathrm{eV}$ and reached a steady state value of $35 \mathrm{eV}$. The density was about $6^{\times} 10^{18} \mathrm{~m}^{-3}$. The point to emphasis from Fig. 9 is that the FRC lives as long as the RMF is on.

\section{DISCUSSIONS}

It is anticipated from a self-consistently study of FRC equilibrium constraints and RMF drive physics that the efficiency of current drive scales inversely with the square root of the frequency $[5,6]$. For this reason the resonant frequency is being lowered to slightly less than half of what it is now. To achieve this lower frequency, the capacitance in the tank circuit is being increased to about 5 times its present value. An additional change involves modifying the program of the waveform generator so that pulses of about $20 \mathrm{~ms}$ will be possible. Along with longer shot duration, an increase in the number of capacitors hooked up to the main capacitor bank will be necessary. 


\section{ACKNOWLEDGMENTS}

The authors would like to thank the Office of Fusion Energy for funding this research. Furthermore, the authors would like to express their gratitude to the following people at RPPL: Bob Brooks, Jon Hayward, Scott Kimball, Mark Kostora, Jim Lynch, and John Slough and the following people at LANL: Hank Alvestad, Kathy Barela, Daniel Begay, Adam Herrera, Patricia Lujan and Dave Miera.

[1] A.L. Hoffman, Physics of Plasmas 5, 979, (1998).

[2] I.R. Jones, Physics of Plasmas 6, 1950, (1999).

[3] I.R. Jones, Technical Report No. FUPH-R-151, NTIS-PB85-133858, Flinders University, Adelaide (1979). Copies are available from the National Technical Information Service, Springfield, VA.

[4] J.T. Slough and K.E. Miller, Review of Scientific Instruments 71, 3210, (2000).

[5] A.L. Hoffman et al., Nuclear Fusion 40, 1523, (2000).

[6] A.L. Hoffman et al., Proc. 18th IAEA Fusion Energy Conference, Sorrento, Italy, 4-10 October 2000. 


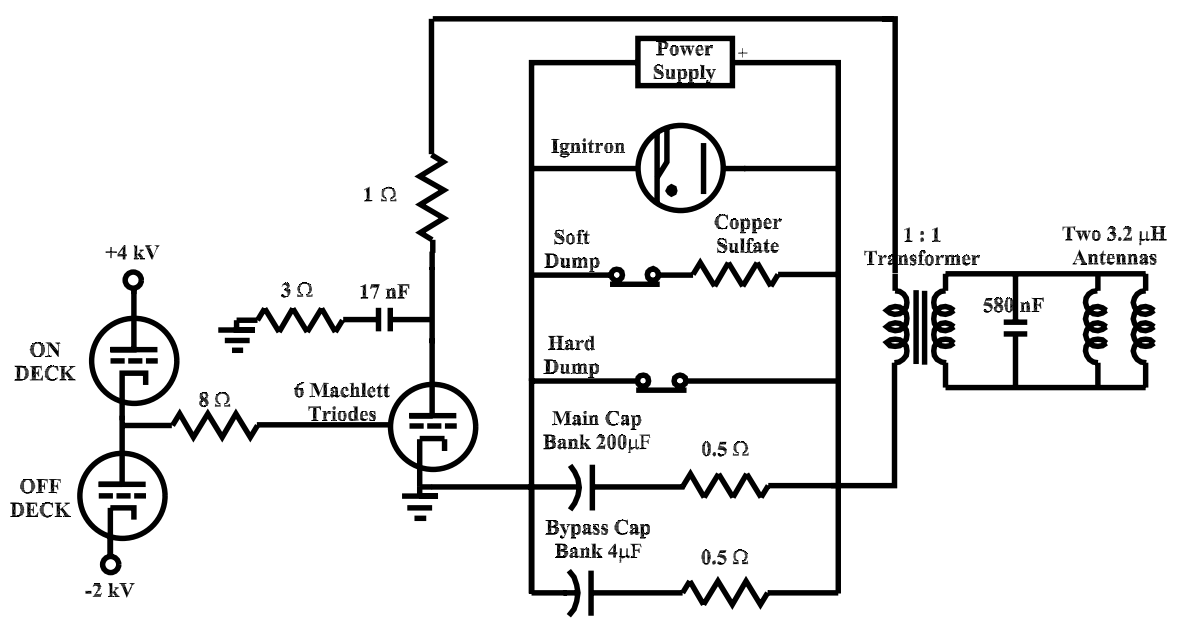

Fig. 1: Simplified circuit diagram of one half of the oscillator system. 


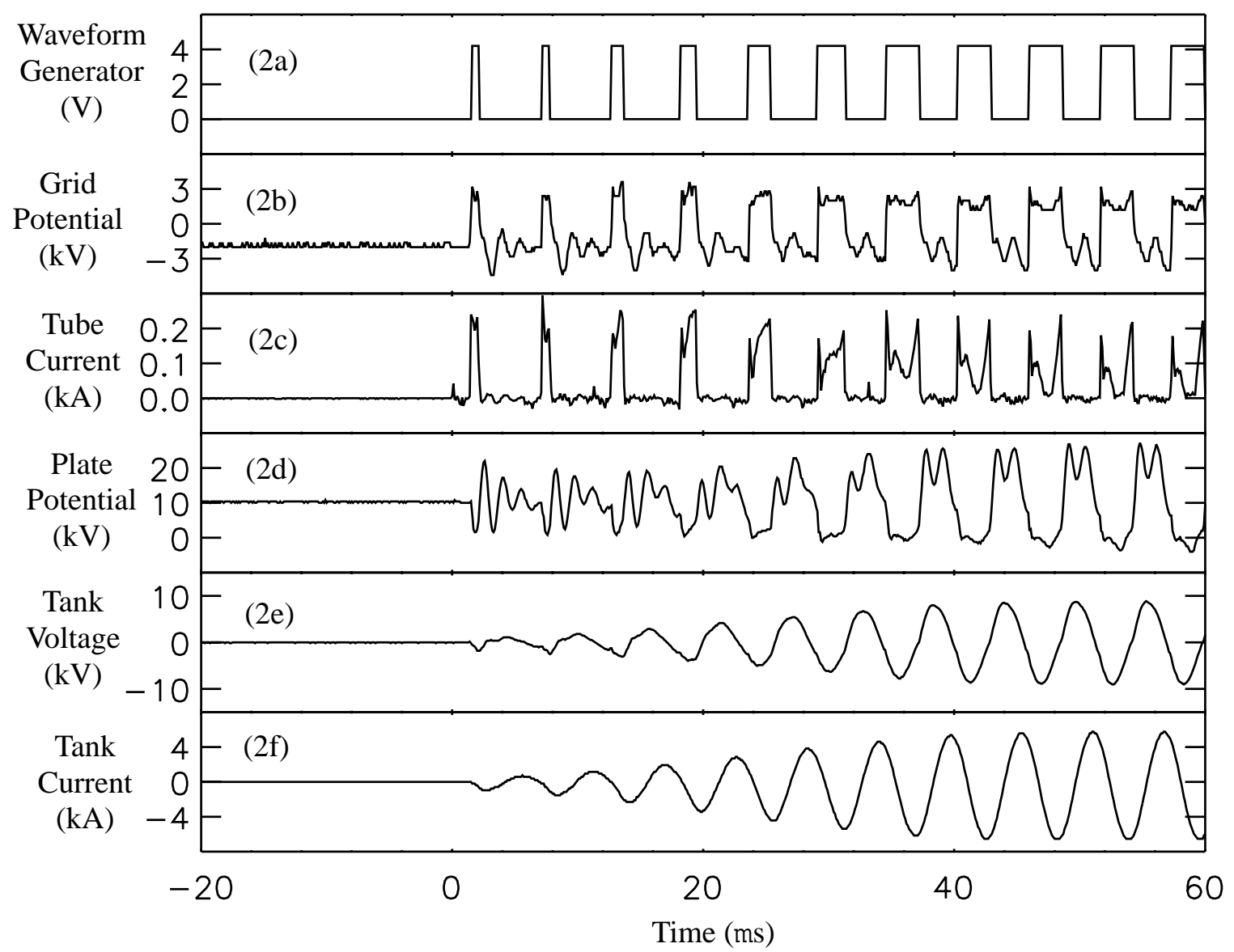

Fig. 2: Voltage and current measurements from various locations on the vertical side of the oscillator system for a $10 \mathrm{kV}$ bank potential and no load: (a) waveform generator output, (b) grid potential, (c) tube current, (d) plate potential, (e) tank circuit voltage, (f) tank circuit voltage. 


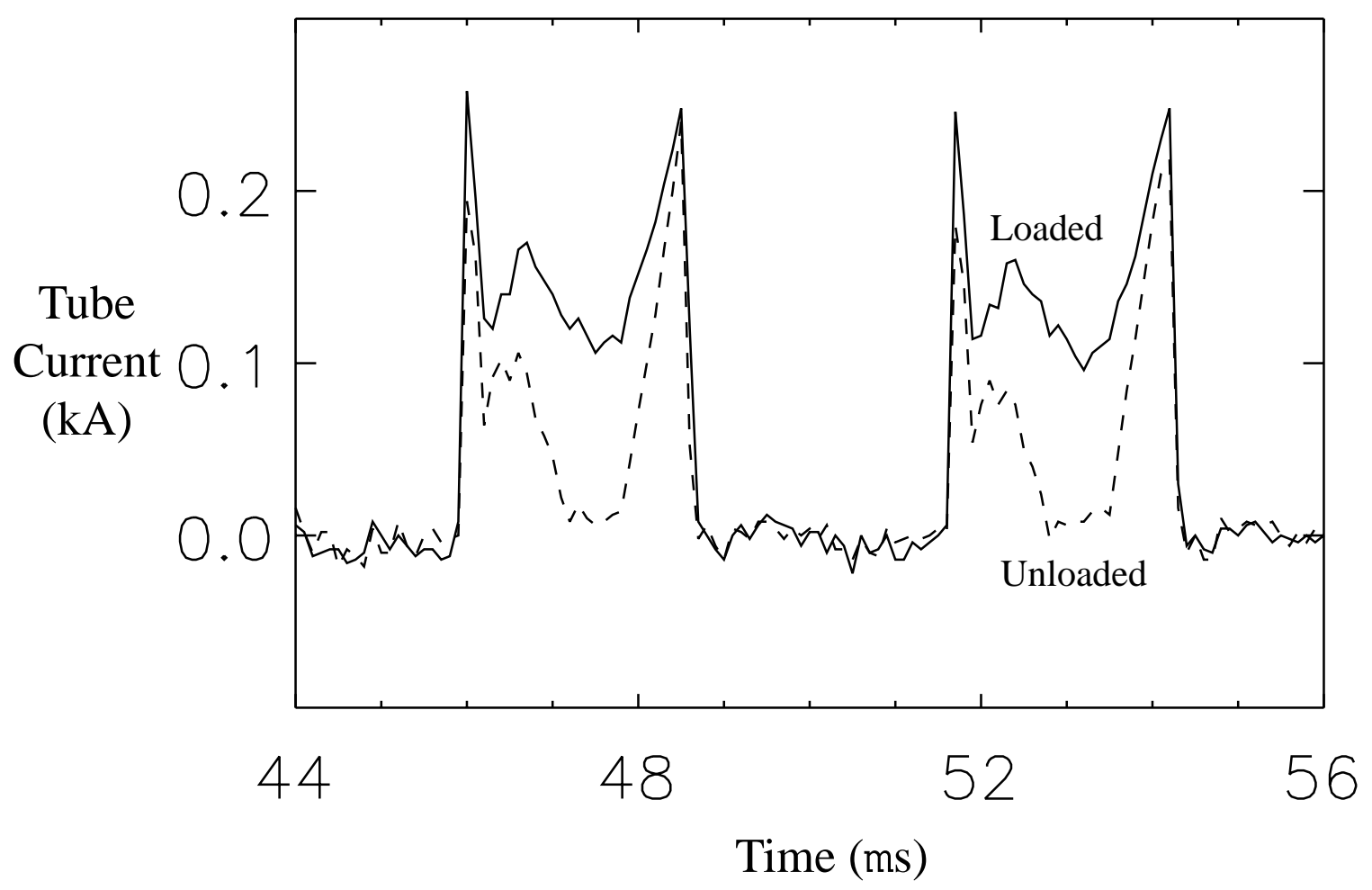

Fig 3: Comparison of the tube current for an unloaded and a loaded case. A resistance of $20 \Omega$ was connected in parallel with vertical antennas for the loaded case.

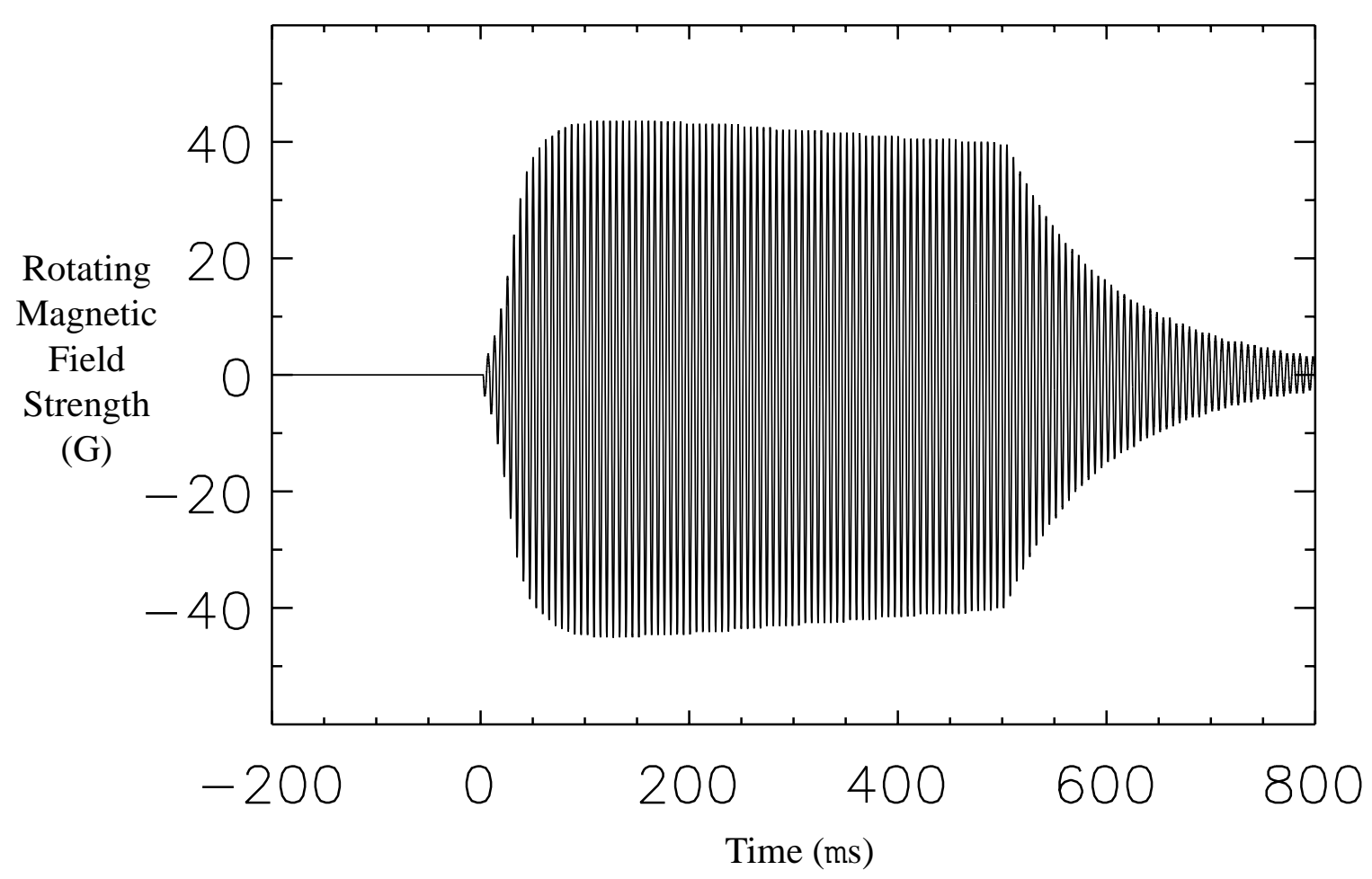

Fig. 4: The RMF as a function of time. 


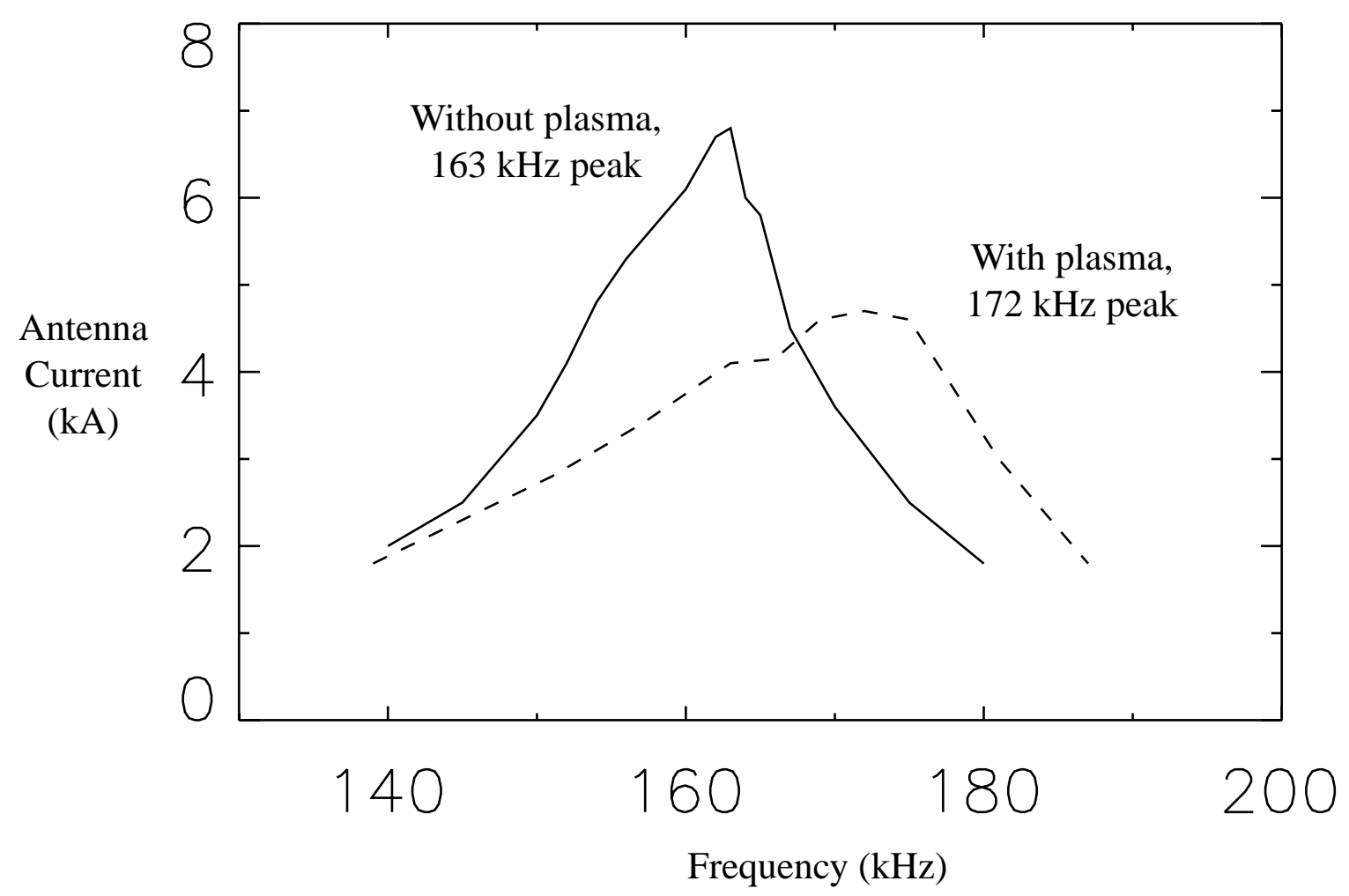

Fig. 5: The circulating current in the tank circuit as a function of frequency for both an unloaded and a plasma case. 


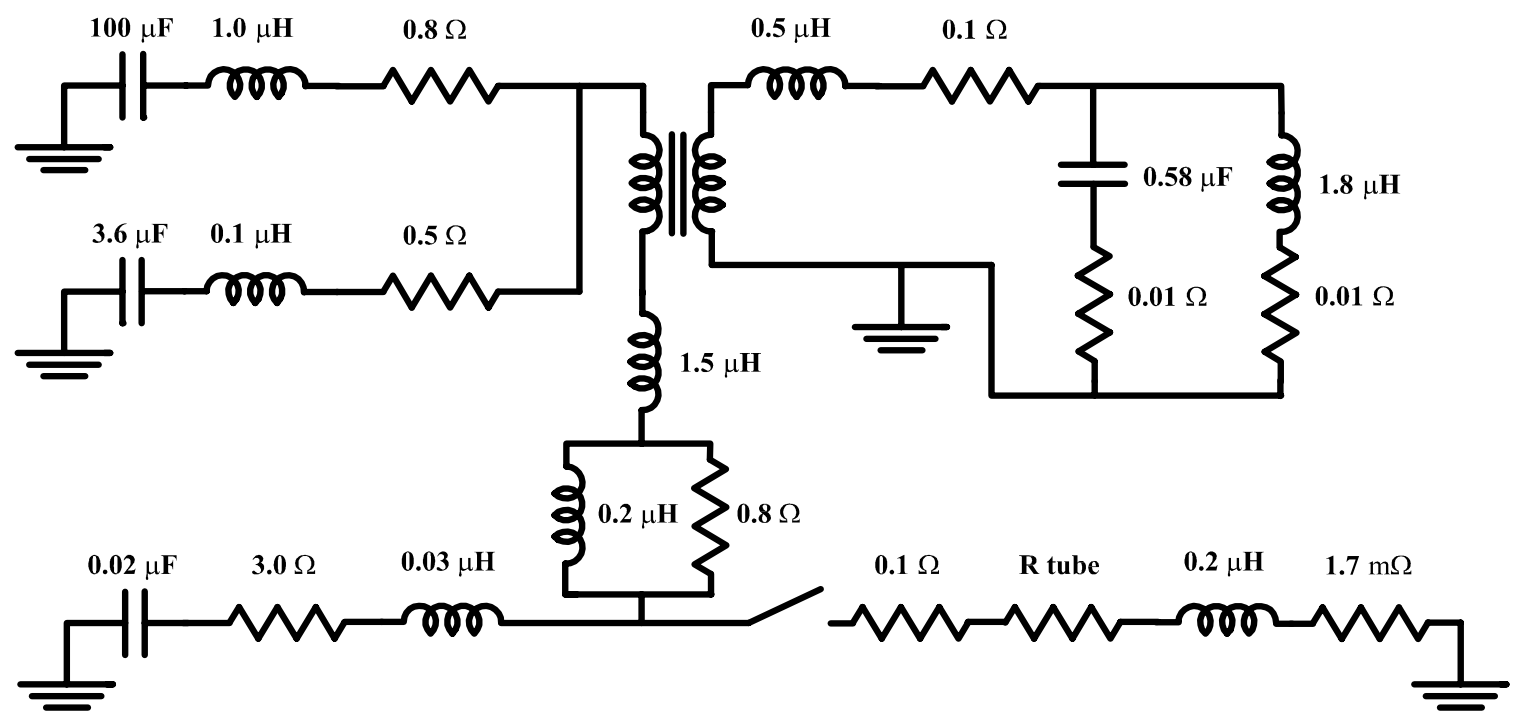

Fig. 6: Schematic of the SPICE model. 


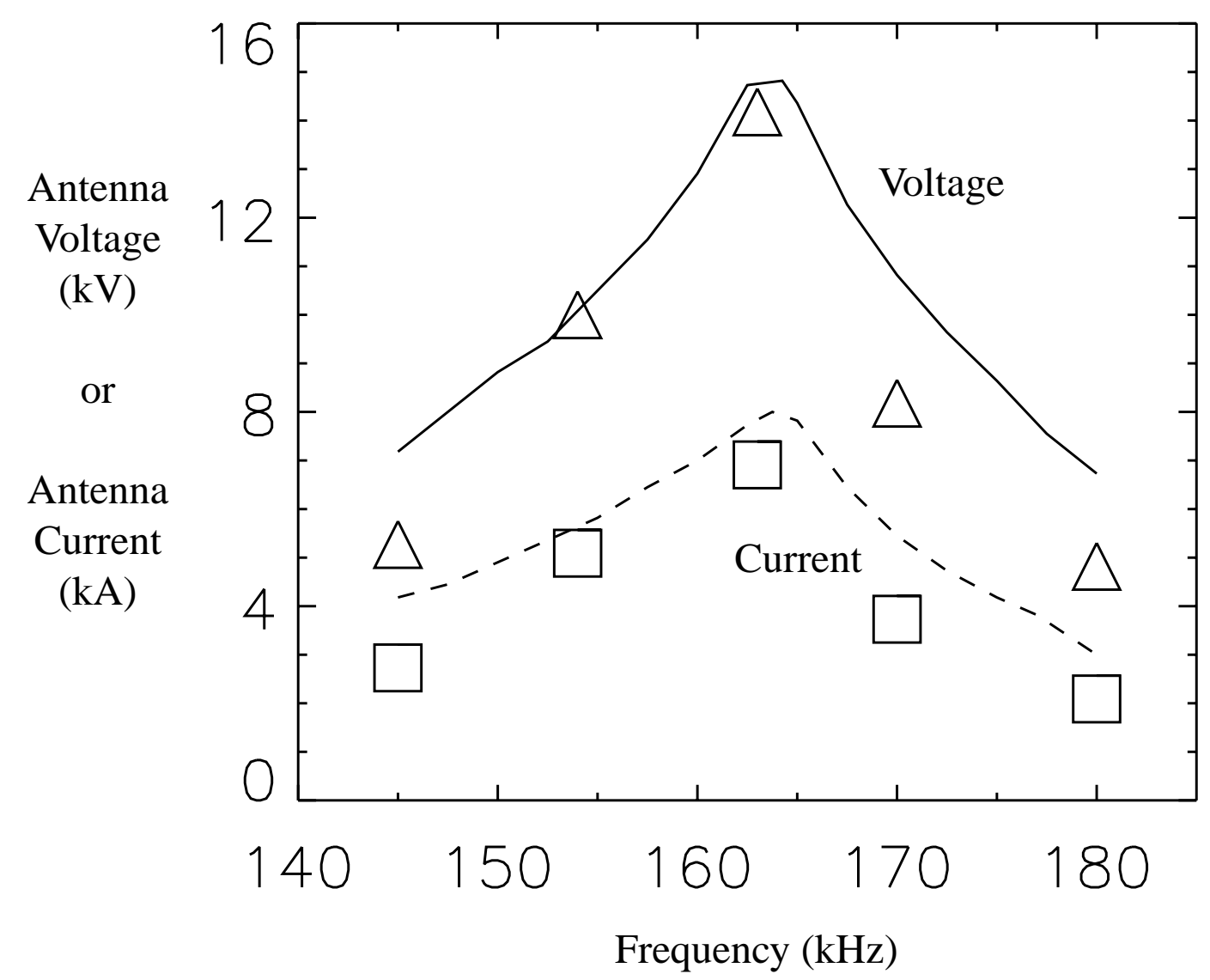

Fig. 7: Comparison of the antenna current and voltage as functions of frequency. The experimentally points are triangles (voltage) and squares (current) and the SPICE model results are solid lines (voltage) and dashed lines (current). 


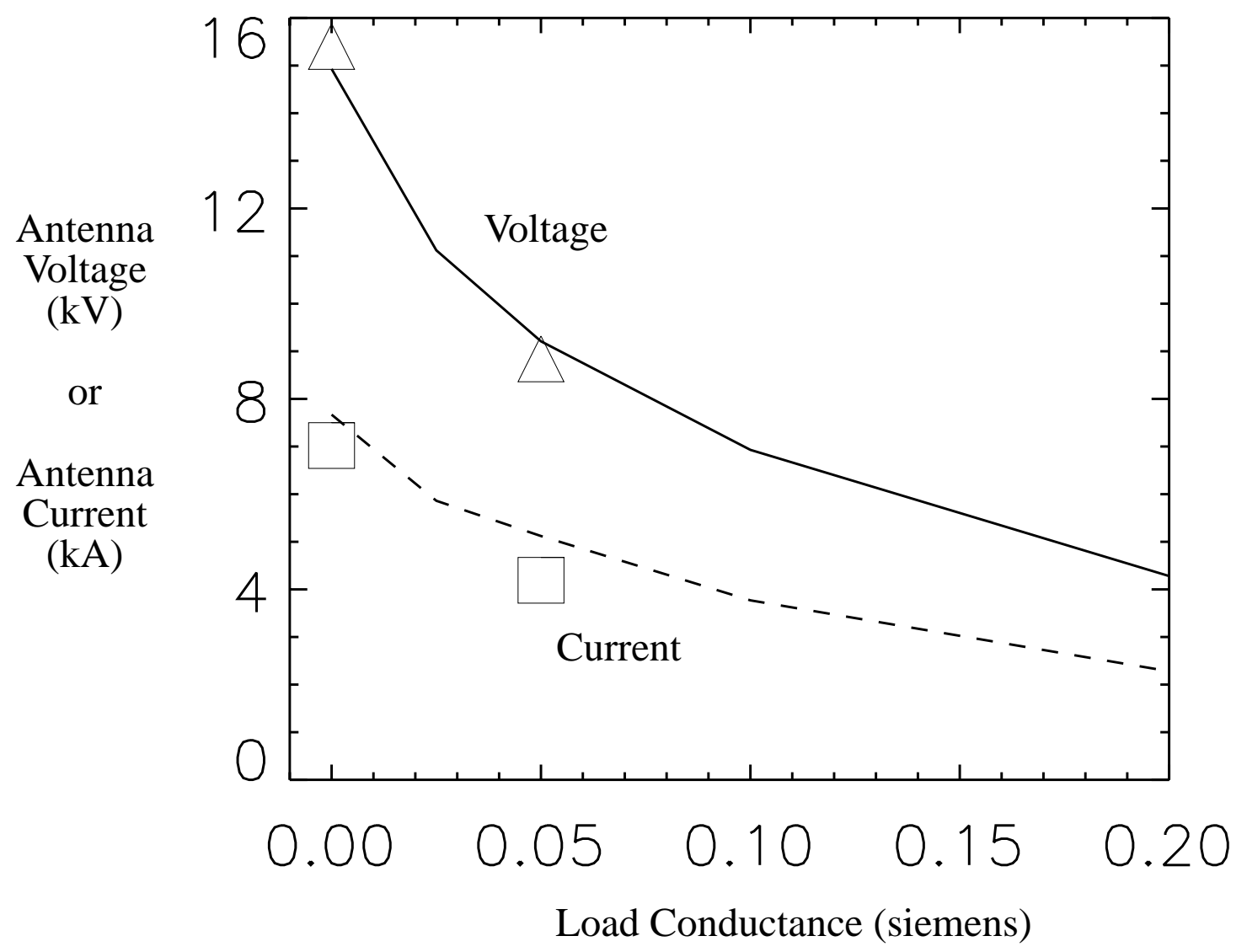

Fig. 8: Comparison of the antenna current and voltage as functions of load conductance. The experimentally points are triangles (voltage) and squares (current) and the SPICE model results are solid lines (voltage) and dashed lines (current). 


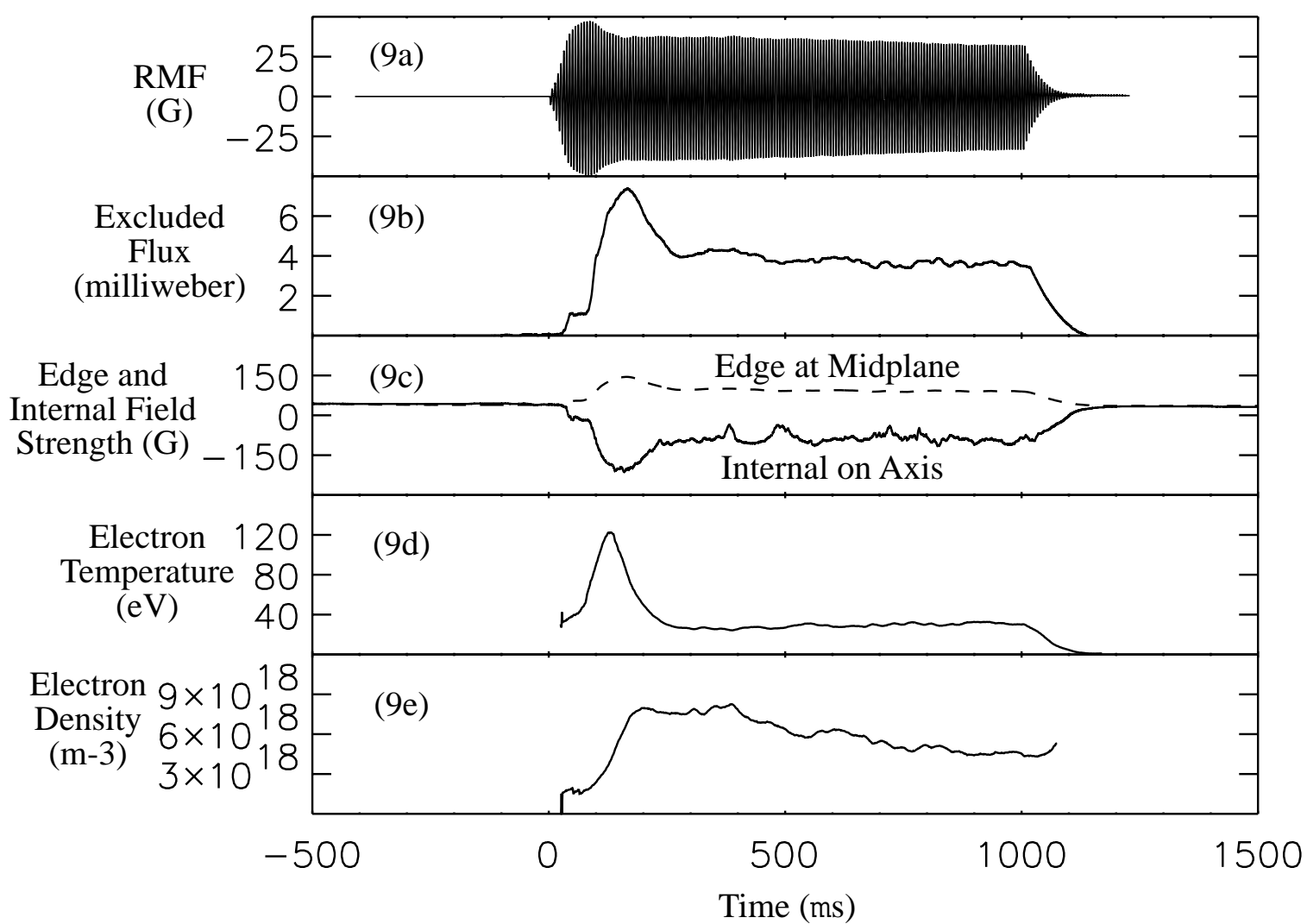

Fig. 9: Data from a typical plasma shot; RMF (12.5 kV bank, 15 psi gas puff) was applied to a pre-ionized plasma: (a) RMF strength, (b) excluded flux, (c) magnetic field strength near the edge of the experimental chamber (dashed line) and magnetic field strength on machine axis (solid line), (d) plasma temperature, (e) plasma density. 